\title{
Crescimento de meloeiro sob estresse salino e doses de potássio ${ }^{1}$
}

\author{
Marcelo T. Gurgel ${ }^{2}$, Cláudio A. Uyeda ${ }^{3}$, Hans R. Gheyi ${ }^{4}$, Fábio H. T. de Oliveira ${ }^{2}$, Pedro D. Fernandes ${ }^{4}$ \& Francisco V. da Silva ${ }^{3}$
}

\begin{abstract}
RESUMO
O Estado do Rio Grande do Norte, em destaque a região da Chapada do Apodi, se destaca na produção e exportação de melão no País, em regime de irrigação, devido à distribuição pluvial baixa e irregular. A Chapada do Apodi possui dois aqüíferos subterrâneos; a do lençol menos profundo de alta salinidade, porém com menor custo de bombeamento, ocorrendo o contrário com o de maior profundidade. Objetivou-se, ante o exposto, avaliar o efeito de duas águas de salinidades diferentes $\left(0,52\right.$ e 2,41 dS $\left.\mathrm{m}^{-1}\right)$, combinadas com cinco doses de $\mathrm{K}_{2} \mathrm{O}\left(218,273,328,383\right.$ e $\left.438 \mathrm{~kg} \mathrm{ha}^{-1}\right)$ sobre $\mathrm{o}$ crescimento do meloeiro (Cucumis melo L.), cultivar Goldex, utilizando-se do delineamento de blocos ao acaso, com parcelas subdivididas; amostras de planta foram coletadas aos 21, 28, 35, 49 e 63 dias após a semeadura, determinandose a fitomassa seca das plantas, estas separadas em ramos (caule + folhas), flores e frutos; avaliou-se, também, a taxa de crescimento absoluto e relativo e a produção de frutos. Em geral, o crescimento do melão foi favorecido com o uso de água mais salina; a taxa de crescimento absoluto foi máxima entre 35 e 49 dias após a semeadura. Obteve-se maior produção de fitomassa total com $438 \mathrm{~kg} \mathrm{ha}^{-1}$ de $\mathrm{K}_{2} \mathrm{O}$ e uso de água mais salina, ao final do ciclo.
\end{abstract}

Palavras-chave: Cucumis melo L, salinidade, fertigação potássica, fitomassa

\section{Growth of melon cultivated under saline stress and potassium doses}

\begin{abstract}
The region of 'Chapada do Apodi', in the State of Rio Grande do Norte, stands out in Brazilian production and exportation of melon. This region possesses two aquiffers, one of lower exploration cost, though of high salinity, another of low salinity, with higher cost and limited use. This work was carried out with the objective to evaluate the effect of waters of different salinities combined with five doses of $\mathrm{K}$ in the dry matter accumulation and productivity of the melon (Cucumis melo L.) cultivar Goldex. The experimental design adopted was split plot in completely randomized blocks. The melon crop was irrigated with low $\left(0.52 \mathrm{dS} \mathrm{m}^{-1}\right)$ and high $\left(2.41 \mathrm{dS} \mathrm{m}^{-1}\right)$ salinity water combined with five doses of $\mathrm{K}_{2} \mathrm{O}(218$, 273, 328, 383 and $438 \mathrm{~kg} \mathrm{ha}^{-1}$ ). Plants were collected at 21, 28, 35, 49 and 63 days after sowing, and were separated in branches (shoot + leaves), flowers and fruits, and the total dry matter of the aerial parts obtained by summation. The absolute and relative growth rates and the production of fruits were also evaluated. The absolute growth rate of the plant was maximum between 35 and 49 days. The highest total biomass was obtained with $438 \mathrm{~kg} \mathrm{ha}^{-1} \mathrm{~K}_{2} \mathrm{O}$ and use of high salinity water at the end of crop cycle.
\end{abstract}

Keywords: Cucumis melo L., salinity, potassium fertigation, dried matter

\footnotetext{
1 Extraida da Tese de Doutorado do primeiro autor, apresentada ao Curso de Recursos Naturais - Centro de Tecnologia e Recursos Naturais da UFCG

2 DCA/UFERSA, BR 110, km 47, CEP 59625-900, Mossoró, RN. Fone: (84) 3315-1741. E-mail: marcelo.tavares@ufersa.edu.br; fabio@ufersa.edu.br

3 Doutorando em Irrigação e Drenagem ESALQ/USP, CEP 13418-900. Piracicaba, SP. Fone (11) 99257-0968. E-mail: cauyeda@yahoo.com.br; valfisio@hotmail.com

${ }^{4}$ UAEA/UFCG, CEP 58109-970, Campina Grande, PB. Fone (83) 3310-1056. E-mail: hans@deag.ufgc.edu.br; pdantas@deag.ufcg.edu.br

${ }^{5}$ UFERSA, Mestrando em Irrigação e Drenagem, CEP 59625-900, Mossoró, RN. Fone (84) 9905-9705. E-mail: agroalmeida@ibeste.com.br
} 


\section{INTRODUÇÃO}

No Brasil, o Estado do Rio Grande do Norte, sobretudo a região do Agropolo Mossoró/Assu, devido às condições edafoclimáticas (solo, temtepatura, umidade relativa do ar, etc.) e à disponibilidade de mananciais de água superficial e subterrânea, tem se destacado no cultivo do meloeiro; nesse estado, a área plantada ocupava, em 2007, aproximadamente 39\% dos 22.048 ha plantados com essa olerícola no Brasil, e a produtividade obtida de $26.920 \mathrm{~kg} \mathrm{ha}^{-1}$ foi superior à do Pais (22.470 $\mathrm{kg} \mathrm{ha}^{-1}$ ) e à do Nordeste (24.510 kg ha-1) (IBGE, 2009).

A principal fonte de água para irrigação no Agropolo Mossoró/Assu é subterrânea, e sua captação é feita do aquífero Arenito - Assu, em poços com cerca de $1.000 \mathrm{~m}$ de profundidade, e do aquífero Calcário Jandaíra, com profundidade em torno de $100 \mathrm{~m}$; atualmente, são mais utilizados os poços que exploram este último aquífero, em decorrência do menor custo de captação. Segundo Oliveira \& Maia (1998), as águas do aquífero Calcário Jandaíra têm concentração de sais relativamente elevada podendo ultrapassar os $2.000 \mathrm{mg} \mathrm{L}^{-1}$ (condutividade elétrica da água - CEa, de aproximadamente $3,0 \mathrm{dS} \mathrm{m}{ }^{-1}$ ), caso em que sua utilização fica condicionada à tolerância das culturas à salinidade e ao manejo da irrigação, com vistas ao controle da salinização dessas áreas.

Os sais, além de afetarem a disponibilidade de água, causam distúrbios nutricionais na planta, dependendo do sal e do genótipo vegetal. A presença de íons em excesso pode impedir a absorção de elementos essenciais ao crescimento da planta, levando ao desbalanceamento nutricional (Tester \& Davenport, 2003).

O meloeiro é uma das cucurbitáceas mais exigentes em relação à adubação. O nutriente extraído do solo em maior quantidade pelo meloeiro é o K (Medeiros et al., 2008). Este nutriente deve influenciar o crescimento do meloeiro, haja vista que as principais funções deste nutriente na planta estão relacionadas com a ativação de muitas enzimas envolvidas na respiração e na fotossíntese, manutenção do equilíbrio iônico e da turgidez das células, controle da abertura e do fechamento dos estômatos, síntese e degradação de amido, transporte de carboidratos no floema, resistência da planta à geada, seca, salinidade do solo e doenças, além de melhorar a qualidade dos frutos. Segundo Filgueira (2000), o K influencia as características qualitativas do melão, pois é indispensável para a formação e amadurecimento dos frutos e para o aumento da textura dos mesmos.

Em vários trabalhos conduzidos na região do Agropólo Mossoró-Assu foram avaliados os efeitos da salinidade da água de irrigação no crescimento, produção e qualidade póscolheita do meloeiro (Dutra et al., 2000; Alencar et al., 2003; Morais et al., 2004; Uyeda et al., 2005; Porto Filho et al., 2006a, 2006b; Queiroga et al., 2006; Silva Júnior et al., 2007; Giehl et al., 2008).

Mais recentemente, Gurgel et al. (2005) mostraram que é viável, economicamente, produzir melões da cultivar Orange Flesh, utilizando água salina $\left(\mathrm{CEa}=3,02 \mathrm{dS} \mathrm{m}^{-1}\right)$, mas para a cultivar Goldex só houve viabilidade econômica quan- do essa cultivar foi irrigada com água de maior salinidade $\left(\mathrm{CEa}=2,4 \mathrm{dS} \mathrm{m}^{-1}\right)$ e adubada com dose mais elevada de $\mathrm{K}_{2} \mathrm{O}$. Diante do exposto, justifica-se a realização de trabalhos para avaliar a possibilidade do aumento da dose de K na adubação e mitigar os efeitos prejudiciais da salinidade em cultivares de meloeiro sensíveis à salinidade da água de irrigação, como a cultivar Goldex.

Neste contexto, se propôs com o presente estudo, avaliar os efeitos de alta e baixa salinidade associada a diferentes doses de $\mathrm{K}_{2} \mathrm{O}$, sobre o crescimento da cultivar de meloeiro Goldex.

\section{MATERIAL E MÉTODOS}

O experimento foi conduzido de setembro a novembro de 2004, na Fazenda Santa Júlia Agrocomercial Exportadora de Frutas Tropicais Ltda., a 8 km a oeste do km 25 da BR 304 e a $20 \mathrm{~km}$ da sede do município de Mossoró, RN, uma das principais regiões produtora de melões do Brasil.

Segundo a classificação de Gaussen, o bioclima da região é 4ath, tropical quente de seca acentuada, com índice xerotérmico entre 200 e 150, e 7 a 8 meses secos. De acordo com a classificação de Köppen, o clima de Mossoró é do tipo BSwh', isto é, seco, muito quente e com estação chuvosa no verão atrasando-se para o outono.

O solo da área experimental é um Argissolo VermelhoAmarelo, com os seguintes atributos, obtidos em análises físico-químicas, realizadas em amostras coletadas na camada 0-20 cm: $18 \%$ de argila; $\mathrm{pH}=7,1$; teores de $\mathrm{P}, \mathrm{Ca}^{2+}$, $\mathrm{Mg}^{2+}, \mathrm{K}^{+}$e $\mathrm{Na}^{+}$iguais a $41 \mathrm{mg} \mathrm{dm}^{-3}, 3,95,1,45,0,23$ e $0,03 \mathrm{cmol}_{\mathrm{C}} \mathrm{dm}^{-3}$, respectivamente, determinados através de metodologias recomendadas pela EMBRAPA (1999).

Estudaram-se os efeitos do uso de águas de baixa $\left(\mathrm{CEa}=0,52 \mathrm{dS} \mathrm{m}^{-1}\right)$ e alta $\left(\mathrm{CEa}=2,41 \mathrm{dS} \mathrm{m}^{-1}\right)$ condutividade elétrica (CEa), associadas a cinco doses de $\mathrm{K}_{2} \mathrm{O}\left(\mathrm{K}_{1}=218\right.$, $\mathrm{K}_{2}=273, \mathrm{~K}_{3}=328, \mathrm{~K}_{4}=383$ e $\mathrm{K}_{5}=438 \mathrm{~kg} \mathrm{ha}^{-1}$ ), sobre $\mathrm{o}$ crescimento do meloeiro (cv Goldex). O delineamento experimental utilizado foi o de blocos ao acaso, em parcelas subdivididas (split plot), com quatro repetições, totalizando 40 sub-parcelas. Cada subparcela teve uma área de $36 \mathrm{~m}^{2}$ (6 6 $6 \mathrm{~m}$ ), formada de três fileiras de plantas espaçadas $2 \mathrm{~m}$. Os tratamentos correspondentes ao fator salinidade da água de irrigação (CEa) foram distribuídos nas parcelas, enquanto os relativos às doses de $\mathrm{K}_{2} \mathrm{O}$ foram aplicadas nas subparcelas.

Na adubação de fundação se aplicaram 47,76, 179,10 e $119,40 \mathrm{~kg} \mathrm{ha}^{-1}$ de $\mathrm{N}, \mathrm{P}_{2} \mathrm{O}_{5}$ e $\mathrm{K}_{2} \mathrm{O}$, respectivamente e, em cobertura, respectivamente, 72,75, 83,22, 58,13 e 3,13 kg ha-1 de $\mathrm{N}, \mathrm{P}_{2} \mathrm{O}_{5}$, S e $\mathrm{MgO}$; enquanto para $\mathrm{K}_{2} \mathrm{O}$ foram 99, 154, 209, 264 e $319 \mathrm{~kg} \mathrm{ha}^{-1}$ para $\mathrm{K}_{1}, \mathrm{~K}_{2}, \mathrm{~K}_{3}, \mathrm{~K}_{4}$ e $\mathrm{K}_{5}$, respectivamente, realizadas a partir do décimo dia após a semeadura. Os adubos aplicados foram: fosfato monoamônico MAP (adubação de fundação), uréia, nitrato de potássio, sulfato de potássio, sulfato de magnésio, ácido fosfórico, CAB2 e Mastermin (adubos foliares).

A irrigação foi por gotejamento, utilizando-se de dois sistemas independentes, para aplicar os diferentes níveis de salinidade da água de irrigação evitando-se, assim, a mistura das 
águas; esses sistemas constaram de duas caixas de fibra de vidro com capacidade de 5.000 L e dois conjuntos motobomba; para evitar e/ou diminuir os problemas fitossanitários, fezse, sistematicamente, o monitoramento da ocorrência de pragas e doenças, sendo adotado o controle fitossanitário normalmente utilizado na Fazenda Santa Júlia.

Para o estudo de crescimento considerou-se apenas o acúmulo de matéria seca da parte aérea e se coletou aos 21, 28, 35, 49 e 63 dias após a semeadura (DAS), uma planta em uma das fileiras da extremidade de cada subparcela; as plantas foram subdivididas em caule e folhas (ramos), flores e frutos, a partir dos quais se determinou a fitomassa seca total da parte aérea (FST).

Calcularam-se, com base na FST, as taxas de crescimento absoluto (TCA) aos 21, 28, 35, 49 e 63 dias após a semeadura (DAS) e as taxas de crescimento relativo (TCR) entre os períodos de 21-28, 29-35, 36-49 e 50-63 DAS, através das fórmulas sugeridas por Benincasa (1988):

$$
\mathrm{TCA}=\frac{\mathrm{FST}_{2}-\mathrm{FST}_{1}}{\mathrm{t}_{2}-\mathrm{t}_{1}} \quad\left(\mathrm{~g} \mathrm{~d}^{-1}\right)
$$

donde:

$\mathrm{FST}_{1}$ - fitomassa seca total da parte aérea, em g, no tempo $t_{1}$, em dias

$\mathrm{FST}_{2}$ - fitomassa seca total da parte aérea, em g, no tempo $t_{2}$, em dias

$$
\mathrm{TCR}=\frac{\mathrm{TCA}}{\text { Fitomassa seca inicial }} \quad\left(\mathrm{g} \mathrm{g}^{-1} \mathrm{~d}^{-1}\right)
$$

Os resultados obtidos foram submetidos a análise de variância simples e análise de regressão polinomial (Ferreira, 2000). Em todo o procedimento de cálculos estatísticos se empregou o software SISVAR.

\section{RESULTADOS E DISCUSSÃO}

\section{Acúmulo de matéria seca}

Aos 21 dias após a semeadura (DAS), constatou-se diferença na fitomassa seca total da parte aérea (FST), entre as qualidades de água utilizadas na irrigação (Tabela 1A); nessa época, a FST era constituída apenas pela fitomassa seca dos ramos, por não haver, ainda, flores nem frutos.

Apesar de o fator $\mathrm{K}_{2} \mathrm{O}$ e a interação 'salinidade x $\mathrm{K}_{2} \mathrm{O}$ ' (Tabela 1A) não terem sido significativos, constatou-se tendência de aumento da FST nas doses mais altas de potássio, quando as plantas foram irrigadas com água mais salina $\left(\mathrm{CEa}=2,41 \mathrm{dS} \mathrm{m}^{-1}\right)$, ao contrário da FST formada com água de baixa CEa $\left(0,52 \mathrm{dS} \mathrm{m}^{-1}\right)$ que se reduziu com o aumento das doses de potássio; na dose de $438 \mathrm{~kg} \mathrm{ha}^{-1}$ de $\mathrm{K}_{2} \mathrm{O}$, a fitomassa foi aproximadamente $30 \%$ maior com o uso de água salina, em comparação com a obtida com água de baixa salinidade.

Aos 28 DAS, começou a haver contribuição das flores na FST, continuando a fitomassa seca dos ramos a ter a maior contribuição na matéria seca total, em todas as doses estudadas de $\mathrm{K}_{2} \mathrm{O}$ (Tabela $1 \mathrm{~B}$ ); além de efeito significativo dos
Tabela 1. Valores médios (g planta ${ }^{-1}$ ) de fitomassa seca total da parte aérea (FST), das flores (FSFL), frutos (FSFR) e ramos (FSR) da cultivar Goldex irrigada com águas de baixa e alta salinidade (CEa) submetida a doses de $\mathrm{K}_{2} \mathrm{O}$ em diferentes épocas

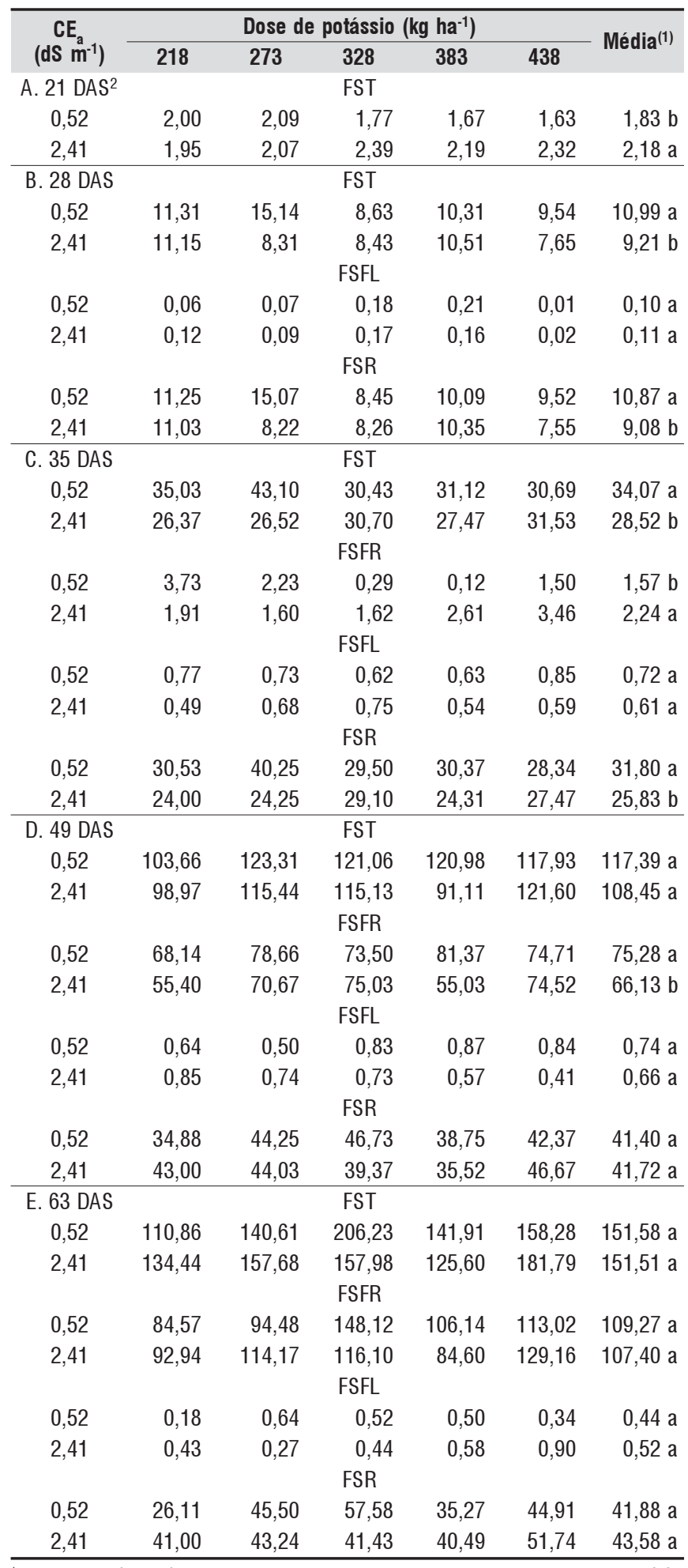

1 Para cada variável, médias seguidas de mesma letra não diferem entre si pelo teste $F$ a $p<0,05$; ${ }^{2}$ DAS - Dias após a semeadura

fatores considerados isoladamente, foi também significativa a interação entre salinidade e doses de $\mathrm{K}_{2} \mathrm{O}$ para as variáveis FST e FSR $(\mathrm{p}<0,01)$ e para a fitomassa seca das flores $($ FSFL) $(\mathrm{p}<0,05)$. 
Ao se confrontar as médias das fitomassas em relação à salinidade e às doses de $\mathrm{K}_{2} \mathrm{O}$, verifica-se recuperação da média de FST com uso da água de $\mathrm{CEa}=0,52 \mathrm{dS} \mathrm{m}^{-1}$ em relação aos 21 DAS que nessa época, ultrapassou a FST obtida com água de maior salinidade, significando que durante este período a cultura é sensível à salinidade da água; assim, através desses valores a solução para o emprego da água salina (2,41 dS m-1) seria aplicá-la após a fase inicial de crescimento da cultura, concordando com Porto Filho et al. (2006b).

Quanto ao estudo de regressão polinomial (Tabela 2B), encontrou-se efeito significativo $(p<0,01)$ para as doses de $\mathrm{K}_{2} \mathrm{O}$ em cada nível de salinidade, para todas as variáveis estudadas aos 28 DAS enquanto a regressão de terceiro grau

Tabela 2. Regressões referentes à fitomassa seca da parte aérea total (FST), das flores (FSFL), frutos (FSFR) e ramos (FSR) para cultivar Goldex irrigada com águas de baixa e alta salinidade (CEa) e submetida a doses de $\mathrm{K}_{2} \mathrm{O}$ em diferentes épocas

\begin{tabular}{|c|c|c|}
\hline $\begin{array}{l}C E_{a} \\
\left(d S m^{-1}\right)\end{array}$ & Regressão & $\mathbf{R}^{2}$ \\
\hline \multicolumn{3}{|c|}{ A. $21 \mathrm{DAS}^{1}$} \\
\hline 0,52 & Nenhum modelo se ajustou aos dados de FST & \\
\hline 2,41 & Nenhum modelo se ajustou aos dados de FST & \\
\hline \multicolumn{3}{|c|}{ B. 28 DAS } \\
\hline 0,52 & $\mathrm{FST}=4 \mathrm{E}-6 \mathrm{x}^{3}-0,004 \mathrm{x}^{2}+1,235 \mathrm{x}-112,553$ & $0,52^{\star *}$ \\
\hline 2,41 & $\mathrm{FST}=-4 \mathrm{E}-6 \mathrm{x}^{3}+0,004 \mathrm{x}^{2}-1,27 \mathrm{x}+143,251$ & $0,95^{\star *}$ \\
\hline 0,52 & $\mathrm{FSFL}=-2 \mathrm{E}-7 \mathrm{x}^{3}+0,0002 \mathrm{x}^{2}-0,0439 \mathrm{x}+4,170$ & $0,99 * *$ \\
\hline 2,41 & $\mathrm{FSFL}=-1 \mathrm{E}-7 \mathrm{x}^{3}+0,0001 \mathrm{x}^{2}-0,0333 \mathrm{x}+3,3137$ & 0,97 ** \\
\hline 0,52 & $\mathrm{FSR}=4 \mathrm{E} 06 \mathrm{x}^{3}-0,0041 \mathrm{x}^{2}+1,2791 \mathrm{x}-116,72$ & $0,53 * *$ \\
\hline 2,41 & $\mathrm{FSR}=-4 \mathrm{E}-6 \mathrm{x}^{3}+0,0039 \mathrm{x}^{2}-1,2535 \mathrm{x}+140,79$ & $0,94^{* *}$ \\
\hline \multicolumn{3}{|c|}{ C. 35 DAS } \\
\hline 0,52 & $\mathrm{FST}=1 \mathrm{E}-5 \mathrm{x}^{3}-0,0098 \mathrm{x}^{2}+3,0893 \mathrm{x}-275,93$ & $0,53 * *$ \\
\hline 2,41 & $\mathrm{FST}=0,02 \mathrm{x}+21,79$ & $0,54^{*}$ \\
\hline 0,52 & FSFR $=1 E-6 x^{3}-0,0008 x^{2}+0,184 x-8,3793$ & 0,99 ** \\
\hline 2,41 & FSFR $=8 E-5 x^{2}-0,0435 x+7,6802$ & $0,97 * *$ \\
\hline 0,52 & $\mathrm{FSFL}=2 \mathrm{E}-5 \mathrm{x}^{2}-0,0098 \mathrm{x}+2,2261$ & $0,78^{* *}$ \\
\hline 2,41 & $\begin{array}{l}F S F L=-1 E-5 x^{2}+0,0087 x-0,7594 \\
F S R\end{array}$ & $0,52^{\star *}$ \\
\hline 0,52 & $F S R=9 E-6 x^{3}-0,0089 x^{2}+2,9082 x-269,05$ & 0,66 ** \\
\hline 2,41 & Nenhum modelo se ajustou aos dados de FSR & \\
\hline \multicolumn{3}{|c|}{ D. 49 DAS } \\
\hline 0,52 & Regressão não significativa & \\
\hline 2,41 & $\mathrm{FST}=4 \mathrm{E}-5 \mathrm{x}^{3}-0,035 \mathrm{x}^{2}+11,128 \mathrm{x}-1033,2$ & $0,84^{* *}$ \\
\hline 0,52 & Regressão não significativa & \\
\hline 2,41 & $F S F R=3 E-5 x^{3}-0,0252 x^{2}+8,1781 x-791,41$ & $0,79 * *$ \\
\hline 0,52 & Regressão não significativa & \\
\hline 2,41 & $\mathrm{FSFL}=-0,0019 \mathrm{x}+1,28$ & $0,93^{*}$ \\
\hline 0,52 & Nenhum modelo se ajustou aos dados de FSR & \\
\hline 2,41 & Nenhum modelo se ajustou aos dados de FSR & \\
\hline \multicolumn{3}{|c|}{ E. 63 DAS } \\
\hline 0,52 & $\mathrm{FST}=-0,0037 x^{2}+2,602 x-281,43$ & $0,55^{\star *}$ \\
\hline 2,41 & $\mathrm{FST}=6 \mathrm{E}-5 \mathrm{x}^{3}-0,0541 \mathrm{x}^{2}+17,033 \mathrm{x}-1586,8$ & $0,88^{* *}$ \\
\hline 0,52 & FSFR $=-0,0024 x^{2}+1,6999 x-175,43$ & 0,51 ** \\
\hline 2,41 & $\mathrm{FSFR}=5 \mathrm{E}-5 \mathrm{x}^{3}-0,0467 \mathrm{x}^{2}+14,799 \mathrm{x}-1410,9$ & $0,84^{* *}$ \\
\hline 0,52 & $\mathrm{FSFL}=-3 \mathrm{E}-5 \mathrm{x}^{2}+0,0177 \mathrm{x}-2,355$ & $0,75^{\star *}$ \\
\hline 2,41 & $\mathrm{FSFL}=0,0023 \mathrm{x}-0,220$ & 0,70 ** \\
\hline 0,52 & $F S R=-0,0013 x^{2}+0,8845 x-103,66$ & 0,50 ** \\
\hline
\end{tabular}

2,41 Nenhum modelo se ajustou aos dados de FSR

${ }^{*} e^{* *}$ - significativo a $p<0,05$ e 0,01 , respectivamente; ${ }^{\text {ns }}$ - Não significativo ${ }^{1}$ DAS - Dias após a semeadura foi a de o maior valor de coeficiente de determinação $\left(\mathrm{R}^{2}\right)$. A dose de $273 \mathrm{~kg} \mathrm{ha}^{-1}$ de $\mathrm{K}_{2} \mathrm{O}$ com uso da água de CEa de $0,52 \mathrm{dS} \mathrm{m}^{-1}$, foi a que resultou em maior acúmulo de matéria seca, aos 28 DAS, com 15,14 g planta-1 e a dose de $438 \mathrm{~kg} \mathrm{ha}^{-1}$ de $\mathrm{K}_{2} \mathrm{O}$, em combinação com a água de maior condutividade elétrica, foi a que mais prejudicou o acúmulo de fitomassa na parte aérea, atingindo 7,65 g planta $^{-1}$ devido, provavelmente, ao fato de o adubo ter contribuído para o agravamento da salinidade da solução do solo.

Farias et al. (2003), trabalhando com a cultivar Gold Mine, aos 26 DAS, submetida a dois níveis de salinidade e seis lâminas de irrigação, obteve fitomassa média de 4,49 g planta $^{-1}$, valor este inferior aos da cultivar estudada neste experimento, o que pode estar relacionado com as diferentes condições de manejo, bem como com as cultivares avaliadas.

A interação entre salinidade e doses de $\mathrm{K}_{2} \mathrm{O}$ aos 35 DAS, para as variáveis FST, FSFL, FSR e fitomassa seca dos frutos (FSFR) foi significativa a p $<0,01$ (Tabela 1C). Em relação à amostragem anterior, nesta fase houve elevação no incremento da FST, passando de 11,31, 15,14, 8,63, 10,31 e 9,54 g planta $^{-1}$, aos 28 DAS, para 35,03, 43,10, 30,43, 31,12 e 30,69 g planta $^{-1}$, aos 35 DAS. Quando as plantas foram irrigadas com a água de $\mathrm{CEa}=0,52 \mathrm{dS} \mathrm{m}^{-1}$ nas doses de 218, 273, 328, 383 e $438 \mathrm{~kg} \mathrm{ha}^{-1}$ de $\mathrm{K}_{2} \mathrm{O}$, respectivamente; entre 28 e 35 DAS o incremento na FSR foi da ordem de 30,97, 26,47, 34,91, 30,10 e 29,70\%, respectivamente, em relação às doses de potássio.

Em geral, enquanto foi pequena a variação nas outras partes da planta, nos frutos se constatou grande incremento de fitomassa seca, entre 28 e 35 DAS, quando o meloeiro foi irrigado com água mais salina $\left(2,41 \mathrm{dS} \mathrm{m}^{-1}\right)$, principalmente nas duas doses mais elevadas de potássio; em relação a dose mais baixa de adubação (218 $\left.\mathrm{kg} \mathrm{ha}^{-1}\right)$, a aplicação de $383 \mathrm{~kg} \mathrm{ha}^{-1}$ de $\mathrm{K}_{2} \mathrm{O}$ resultou em 7,28\% de aumento na FSFR, subindo $44,80 \%$ na dose mais alta de potássio (438 $\mathrm{kg} \mathrm{ha}^{-1}$ de $\mathrm{K}_{2} \mathrm{O}$ ).

$\mathrm{O}$ maior efeito da adubação de $\mathrm{K}_{2} \mathrm{O}$ com o uso de água mais salina, aos $35 \mathrm{DAS}$, pode ser um indicativo de maior tolerância da cultivar Goldex ao sódio nesta fase, coincidindo com o início da frutificação.

Com base nos estudos de regressão, ocorreu efeito significativo para FST, FSFR e FSR $(\mathrm{p}<0,01)$ e para FSFL ( $<<0,05)$ para o menor nível de salinidade; já para o nível de maior salinidade se encontrou efeito significativo apenas em FSFR e FSFL (Tabela 2C). Para a fitomassa seca total da parte aérea (FST), a regressão cúbica foi a que melhor se ajustou aos dados para a água de menor salinidade, e, para a água de maior salinidade, encontrou-se equação linear crescente, constatando-se que, com o aumento das doses de $\mathrm{K}_{2} \mathrm{O}$, os efeitos nocivos da água de alta salinidade $\left(\mathrm{CEa}=2,41 \mathrm{dS} \mathrm{m}^{-1}\right.$ ) diminuiram.

Aos 49 DAS a interação entre salinidade e doses de $\mathrm{K}_{2} \mathrm{O}$ foi significativa a $(\mathrm{p}<0,05)$ para FST e FSFL e $(\mathrm{p}<0,01)$ para FSFR, não havendo significância para a FSR (Tabela 1D). Nesta época, o aumento da FST se deu pelo acréscimo da FSFR representando 64,13 e 60,98\%, respectivamente, quando se utilizou água de condutividade elétrica de 0,52 e 2,41 dS m-1. 
A contribuição da FSFR para a variável FST demonstrou recuperação da FSFR nas doses superiores a $273 \mathrm{~kg} \mathrm{ha}^{-1}$ de $\mathrm{K}_{2} \mathrm{O}$, no nível menos salino pois aos 49 DAS os valores de fitomassa seca dos frutos foram maiores em comparação com os obtidos na menor dose aplicada de $\mathrm{K}_{2} \mathrm{O}$.

Constatou-se acréscimo na fitomassa seca dos ramos, entre 35 e 49 DAS, passando de 31,80 para 41,40 g planta $^{-1}$, no nível de salinidade mais baixo da água de irrigação, e de 25,83 para 41,72 g planta $^{-1}$ no nível mais salino da água. O maior incremento de FSR ocorreu no nível mais alto de CEa $\left(2,41 \mathrm{dS} \mathrm{m}^{-1}\right)$, com 38,09\% de incremento, enquanto na água de $\mathrm{CEa}=0,52 \mathrm{dS} \mathrm{m}^{-1}$ se obteve aumento médio de 23,19\%.

Com base no estudo da regressão (Tabela 2D), não houve significância para as doses de $\mathrm{K}_{2} \mathrm{O}$ nas variáveis de fitomassa, com uso de água de baixa salinidade, entretanto, para o nível mais salino da água se registraram significâncias $(p<0,01)$ para FST, FSFR, e $(p<0,05)$ para fitomassa seca das flores (FSFL).

Para FST e FSFR, na CEa $=2,41 \mathrm{dS} \mathrm{m}^{-1}$, o modelo cúbico foi o melhor ajustado; em relação à FSFL, obteve-se efeito linear decrescente, diminuindo a produção de FSFL com o aumento das doses de $\mathrm{K}_{2} \mathrm{O}$, aos 49 DAS (Tabela 2D).

Quanto ao acúmulo de fitomassa seca, aos 63 DAS nas diferentes partes da planta (Tabela $1 \mathrm{E}$ ), notou-se efeito significativo na interação entre doses de $\mathrm{K}_{2} \mathrm{O}$ e níveis de salinidade da água de irrigação.

Nos frutos, os maiores valores de fitomassa seca, 148,12 e 129,16 g planta $^{-1}$, foram obtidos com águas de 0,52 e $2,41 \mathrm{dS} \mathrm{m}^{-1}$, nas doses 328 e $438 \mathrm{~kg} \mathrm{ha}^{-1}$ de $\mathrm{K}_{2} \mathrm{O}$, respectivamente; os menores valores de 84,57 e 84,60 $\mathrm{g} \mathrm{planta}^{-1}$ foram observados nas doses 218 e $383 \mathrm{~kg} \mathrm{ha}^{-1}$ de $\mathrm{K}_{2} \mathrm{O}$, respectivamente, em condições de baixa e alta salinidade da água de irrigação. Nota-se que no nível salino da água (2,41 dS m $\left.{ }^{-1}\right)$ houve maior acúmulo de fitomassa nos frutos ao se aplicar a dose de $438 \mathrm{~kg} \mathrm{ha}^{-1} \mathrm{~K}_{2} \mathrm{O}$ (Tabela $1 \mathrm{E}$ ).

Com relação à FSR, obtiveram-se valores médios máximos de 57,58 e 51,74 g planta $^{-1}$, com as águas de 0,52 e $2,42 \mathrm{dS} \mathrm{m}^{-1}$, respectivamente, referentes às doses $328 \mathrm{e}$ $438 \mathrm{~kg} \mathrm{ha}^{-1}$ de $\mathrm{K}_{2} \mathrm{O}$, semelhante ao comportamento ocorrido nos frutos, embora nesta variável não tenha havido diferença significativa da fitomassa, entre as doses de $\mathrm{K}$, no maior nível de CEa; ao contrário, quando as plantas foram irrigadas com água de baixa salinidade foi visível a diferença significativa, com valor mínimo de 26,11 g planta $^{-1}$ ao se aplicar a quantidade de $218 \mathrm{~kg} \mathrm{ha}^{-1}$ de $\mathrm{K}_{2} \mathrm{O}$ (Tabela $1 \mathrm{E}$ ).

Para a FST da parte aérea, também se constataram os valores máximos (206,23 e 181,79 g planta $^{-1}$ ) quando foram aplicados 328 e $438 \mathrm{~kg} \mathrm{ha}^{-1}$ de $\mathrm{K}_{2} \mathrm{O}$, respectivamente, em condições de baixa e alta salinidade $\left(0,52\right.$ e 2,41 dS m$\left.{ }^{-1}\right)$, além de valores mínimos de 110,86 e 125,60 g planta $^{-1}$ nas doses de 218 e $383 \mathrm{~kg} \mathrm{ha}^{-1}$ de $\mathrm{K}_{2} \mathrm{O}$, respectivamente, para 0,52 e $2,41 \mathrm{dS} \mathrm{m}^{-1}$.

Percebe-se que, de modo geral, a aplicação de potássio aumentou a fitomassa quando as plantas foram irrigadas com água salina $\left(2,41 \mathrm{dS} \mathrm{m}^{-1}\right)$, principalmente a biomassa dos frutos, os maiores responsáveis pelo acúmulo de fitomassa seca na parte aérea; estes são resultados semelhantes aos obtidos por Medeiros et al. (2008) ao avaliarem o crescimento e acúmulo de N, P e K pelo meloeiro irrigado com água salina. Tal fato pode estar relacionado com a função do potássio nas plantas, influenciando no peso dos frutos em virtude do seu importante papel na translocação dos carboidratos (Epstein \& Bloom, 2003).

Porto Filho et al. (2006b), avaliando a viabilidade da irrigação do meloeiro com águas salinas ( $\mathrm{S} 1=0,6, \mathrm{~S} 2=1,9$, $\mathrm{S} 3=3,2$ e S4 $=4,5 \mathrm{dS} \mathrm{m}^{-1}$ ), em diferentes fases fenológicas, verificaram que, quanto mais próximo do início do ciclo cultural do meloeiro, o uso de águas salobras na irrigação reduz a produção comercial e total de frutos. Pode-se considerar, neste contexto, que maiores aplicações de potássio na fase inicial podem reduzir ou minimizar os efeitos negativos da salinidade da água de irrigação.

\section{Taxa de crescimento absoluto e relativo}

Na taxa de crescimento absoluto (TCA), aos 21 dias após a semeadura (DAS), foi observado efeito significativo $(\mathrm{p}<0,05)$ apenas entre os níveis salinos da água, sem significância para a interação entre doses de $\mathrm{K}_{2} \mathrm{O}$ e salinidade (Tabela 3). A maior TCA média, em relação à salinidade, nesta época, foi obtida no maior nível salino da água de irrigação $\left(\mathrm{CEa}=2,41 \mathrm{dS} \mathrm{m}^{-1}\right)$, com produção de

Tabela 3. Valores médios de taxas de crescimento absoluto (TCA) e relativo (TCR) da cultivar Goldex de meloeiro irrigada com águas de baixa e alta salinidade (CEa) e submetida a doses de $\mathrm{K}_{2} \mathrm{O}$

\begin{tabular}{|c|c|c|c|c|c|c|}
\hline \multirow{2}{*}{$\begin{array}{c}C E_{a} \\
\left(d S \mathrm{~m}^{-1}\right)\end{array}$} & \multicolumn{5}{|c|}{ Dose de potássio $\left(\mathrm{kg} \mathrm{ha}^{-1}\right)$} & \multirow{2}{*}{ Média $^{(1)}$} \\
\hline & 218 & 273 & 328 & 383 & 438 & \\
\hline $21 \mathrm{DAS}^{2}$ & \multicolumn{6}{|c|}{ TCA $\left(\mathrm{g} \mathrm{d}^{-1}\right)$} \\
\hline 0,52 & 0,097 & 0,1000 & 0,085 & 0,080 & 0,077 & $0,088 \mathrm{~b}$ \\
\hline 2,41 & 0,092 & 0,1000 & 0,112 & 0,102 & 0,112 & $0,104 \mathrm{a}$ \\
\hline \multicolumn{7}{|l|}{28 DAS } \\
\hline 0,52 & 1,327 & 1,865 & 0,982 & 1,235 & 1,130 & $1,308 \mathrm{a}$ \\
\hline 2,41 & 1,315 & 0,890 & 0,862 & 1,187 & 0,762 & $1,003 b$ \\
\hline \multicolumn{7}{|l|}{35 DAS } \\
\hline 0,52 & 3,390 & 3,992 & 3,112 & 2,975 & 3,022 & $3,298 \mathrm{a}$ \\
\hline 2,41 & 2,172 & 2,602 & 3,180 & 2,425 & 3,412 & $2,758 \mathrm{~b}$ \\
\hline \multicolumn{7}{|l|}{49 DAS } \\
\hline 0,52 & 4,90 & 5,730 & 6,472 & 6,420 & 6,232 & $5,952 \mathrm{a}$ \\
\hline 2,41 & 5,185 & 6,352 & 6,030 & 4,547 & 6,432 & $5,709 \mathrm{a}$ \\
\hline \multicolumn{7}{|l|}{63 DAS } \\
\hline 0,52 & 0,515 & 1,237 & 6,082 & 1,495 & 2,880 & $2,442 \mathrm{a}$ \\
\hline 2,41 & 2,532 & 3,017 & 3,060 & 2,470 & 4,297 & $3,075 \mathrm{a}$ \\
\hline 21 a 28 DAS & \multicolumn{6}{|c|}{ TCR $\left(\mathrm{g} \mathrm{g}^{-1} \mathrm{~d}^{-1}\right)$} \\
\hline 0,52 & 0,660 & 0,890 & 0,575 & 0,750 & 0,700 & $0,715 \mathrm{a}$ \\
\hline 2,41 & 0,715 & 0,435 & 0,367 & 0,577 & 0,345 & $0,488 \mathrm{~b}$ \\
\hline \multicolumn{7}{|l|}{29 a 35 DAS } \\
\hline 0,52 & 0,300 & 0,262 & 0,362 & 0,295 & 0,322 & $0,308 \mathrm{a}$ \\
\hline 2,41 & 0,195 & 0,317 & 0,392 & 0,230 & 0,450 & $0,317 \mathrm{a}$ \\
\hline \multicolumn{7}{|l|}{36 a 49 DAS } \\
\hline 0,52 & 0,140 & 0,135 & 0,217 & 0,212 & 0,207 & $0,182 \mathrm{~b}$ \\
\hline 2,41 & 0,205 & 0,240 & 0,200 & 0,172 & 0,205 & $0,204 \mathrm{a}$ \\
\hline \multicolumn{7}{|l|}{50 a 63 DAS } \\
\hline 0,52 & 0,005 & 0,010 & 0,047 & 0,012 & 0,022 & $0,019 \mathrm{a}$ \\
\hline 2,41 & 0,025 & 0,027 & 0,025 & 0,025 & 0,035 & $0,027 \mathrm{a}$ \\
\hline
\end{tabular}

(1) Para cada variável, médias seguidas de mesma letra não diferem entre si pelo teste $\mathrm{F}$ a 5 \%; ${ }^{2}$ DAS - Dias após a semeadura 
0,104 $\mathrm{g} \mathrm{d}^{-1}$ de fitomassa, enquanto no menor nível salino $\left(\mathrm{CEa}=0,52 \mathrm{dS} \mathrm{m}^{-1}\right)$, a taxa foi de apenas $0,088 \mathrm{~g} \mathrm{~d}^{-1}$.

A dose de $328 \mathrm{~kg} \mathrm{ha}^{-1}$ de $\mathrm{K}_{2} \mathrm{O}$, combinada com água de alta salinidade, foi a que resultou em maior TCA, com valor de $0,112 \mathrm{~g} \mathrm{~d}^{-1}$, enquanto com a dose de $438 \mathrm{~kg} \mathrm{ha}^{-1} \mathrm{e}$ água de baixa salinidade, se obteve menor TCA, equivalente a $0,077 \mathrm{~g} \mathrm{~d}^{-1}$.

O K $\mathrm{K}^{+}$é o principal nutriente relacionado a funções osmóticas das plantas; conforme Satti \& Lopez (1994), o melhor desempenho de alguns genótipos mantidos sob condições de estresse salino tem sido associado a uma nutrição potássica adequada, porém, o aumento da proporção de $\mathrm{K}^{+}$, em meio contendo $\mathrm{NaCl}$, nem sempre resulta em efeitos benéficos para as plantas, podendo a salinidade provocada por altas concentrações de $\mathrm{K}^{+}$ser, inclusive, mais prejudicial que a provocada por altas concentrações de sódio, o que pode ter ocorrido nesse estudo.

Aos 28 DAS, a TCA foi significativamente afetada pelos fatores (salinidade e doses de potássio), isoladamente, e para a interação entre salinidade e doses de potássio (Tabela 3). Em relação à salinidade, o valor médio da TCA da água de menor CEa foi maior que a TCA na água de alta salinidade, nessa época, justificado pela recuperação da fitomassa seca total da parte aérea, em relação à da data anterior. Maior TCA, aos 28 DAS, foi constatada na dose de $273 \mathrm{~kg} \mathrm{ha}^{-1}$ de $\mathrm{K}_{2} \mathrm{O}$, quando as plantas foram irrigadas com água de $\mathrm{CEa}=0,52 \mathrm{dS} \mathrm{m}^{-1}$, obtendo-se o valor de $1,865 \mathrm{~g} \mathrm{~d}^{-1}$; a maior dose de $\mathrm{K}_{2} \mathrm{O}$, combinada com a água de $\mathrm{CEa}=2,41 \mathrm{dS} \mathrm{m}^{-1}$, foi a mais prejudicial, resultando em uma TCA de $0,762 \mathrm{~g} \mathrm{~d}^{-1}$. Tais resultados refletem a maior sensibilidade da cultura, nessa época, à salinidade.

Com relação ao estudo de regressão (Tabela 4), para o menor nível de salinidade da água e apesar de haver efeitos significativos $(\mathrm{p}<0,01)$ para a regressão polinomial de $1^{\mathrm{a}}$ e $3^{\mathrm{a}}$ ordem, os coeficientes de determinação dessas equações foram muito baixos $\left(\mathrm{R}^{2}<0,50\right)$, em razão da grande dispersão dos dados; já para a água de $\mathrm{CEa}=2,41 \mathrm{dS} \mathrm{m}^{-1}$ houve efeitos significativos $(p<0,01)$ para as regressões linear e cúbica, porém as equações de $3^{a}$ ordem foram as que tiveram maiores $\mathrm{R}^{2}$.

Houve tendência similar entre os valores de TCA, aos 35 e 49 DAS, sendo também maiores quando as plantas foram irrigadas com água de melhor qualidade; nas duas épocas foi significativa a interação entre os fatores.

Aos 35 DAS (Tabela 3), o maior valor de TCA foi 3,992 $\mathrm{g} \mathrm{d}^{-1}$, encontrado com o uso da água de menor salinidade e a dose de $273 \mathrm{~kg} \mathrm{ha}^{-1}$ de $\mathrm{K}_{2} \mathrm{O}$; aos 49 DAS, o maior valor de TCA foi $6,472 \mathrm{~g} \mathrm{~d}^{-1}$ obtido, também, com a água de baixa salinidade, quando a adubação potássica foi na dose de $328 \mathrm{~kg} \mathrm{ha}^{-1}$ de $\mathrm{K}_{2} \mathrm{O}$; irrigando-se as plantas com a água mais salina, o maior valor de TCA $\left(6,432 \mathrm{~g} \mathrm{~d}^{-1}\right)$ foi obtido com a maior dose de $\mathrm{K}_{2} \mathrm{O}$. Analisando-se os dados de TCA, obtidos com a dose de $438 \mathrm{~kg} \mathrm{ha}^{-1}$ de $\mathrm{K}_{2} \mathrm{O}$ e $\mathrm{CEa}=2,41 \mathrm{dS} \mathrm{m}^{-1}$, notou-se recuperação nas datas seguintes, passando de 0,762 aos 28 DAS para 3,714 $\mathrm{g} \mathrm{d}^{-1}$ aos 35 DAS sendo, também, altos nas épocas seguintes de amostragens.

$\mathrm{Na}$ análise de regressão (Tabela 4) para as doses de $\mathrm{K}_{2} \mathrm{O}$ na água de baixa salinidade não se registrou efeito signifi-
Tabela 4. Regressões referentes às taxas de crescimento absoluto (TCA) e relativo (TCR) da cultivar Goldex de meloeiro irrigada com águas de baixa e alta salinidade (CEa) e submetida a doses de $\mathrm{K}_{2} \mathrm{O}$

\begin{tabular}{|c|c|c|}
\hline $\begin{array}{c}C E_{\mathrm{a}} \\
\left(\mathrm{dS} \mathrm{m}^{-1}\right)\end{array}$ & Regressão & $\mathbf{R}^{2}$ \\
\hline $21 \mathrm{DAS}^{1}$ & TCA $\left(\right.$ g dia $\left.^{-1}\right)$ & \\
\hline 0,52 & Nenhum modelo se ajustou aos dados & \\
\hline 2,41 & Nenhum modelo se ajustou aos dados & \\
\hline \multicolumn{3}{|l|}{28 DAS } \\
\hline 0,52 & Nenhum modelo se ajustou aos dados de TCA & \\
\hline 2,41 & $\operatorname{TCA}=-6 \mathrm{E}-07 \mathrm{x}^{3}+0,0006 \mathrm{x}^{2}-0,187 \mathrm{x}+20,67$ & $0,93^{* *}$ \\
\hline \multicolumn{3}{|l|}{25 DAS } \\
\hline 0,52 & Regressão não significativa & \\
\hline 2,41 & TCA $=8 \mathrm{E}-07 \mathrm{x}^{3}-0,0008 \mathrm{x}^{2}+0,2572 \mathrm{x}-24,632$ & $0,73^{*}$ \\
\hline \multicolumn{3}{|l|}{39 DAS } \\
\hline 0,52 & Regressão não significativa & \\
\hline 2,41 & $\mathrm{TCA}=2 \mathrm{E}-06 \mathrm{x}^{3}-0,0024 \mathrm{x}^{2}+0,7572 \mathrm{x}-71,69$ & $0,91^{* *}$ \\
\hline \multicolumn{3}{|l|}{63 DAS } \\
\hline 0,52 & Nenhum modelo se ajustou aos dados de TCR & \\
\hline 2,41 & $\mathrm{TCA}=1 \mathrm{E}-06 \mathrm{x}^{3}-0,0014 \mathrm{x}^{2}+0,4212 \mathrm{x}-39,499$ & $0,93^{*}$ \\
\hline 29 a 35 DAS & TCR $\left(\mathrm{g} \mathrm{g}^{-1} \mathrm{dia}^{-1}\right)$ & \\
\hline 0,52 & Nenhum modelo se ajustou aos dados de TCR & \\
\hline 2,41 & TCR $=-3 \mathrm{E}-07 \mathrm{x}^{3}+0,0003 \mathrm{x}^{2}-0,1094 \mathrm{x}+12,21$ & $0,91^{*}$ \\
\hline \multicolumn{3}{|l|}{29 a 35 DAS } \\
\hline 0,52 & Regressão não significativa & \\
\hline 2,41 & TCR $=2 \mathrm{E}-07 \mathrm{x}^{3}-0,0002 \mathrm{x}^{2}+0,0687 \mathrm{x}-6,910$ & 0,80 ** \\
\hline \multicolumn{3}{|l|}{36 a 49 DAS } \\
\hline 0,52 & $\mathrm{TCR}=0,0004 \mathrm{x}+0,0558$ & $0,66^{\star *}$ \\
\hline 2,41 & Regressão não significativa & \\
\hline \multicolumn{3}{|c|}{50 a 63 DAS } \\
\hline 0,52 & Nenhum modelo se ajustou aos dados de TCR & \\
\hline 2,41 & Regressão não significativa & \\
\hline
\end{tabular}

cativo e, para o maior nível salino, houve efeito para a regressão polinomial de $1^{\mathrm{a}}$ ordem $(\mathrm{p}<0,01)$, aos $35 \mathrm{DAS}$, porém, a equação cúbica foi a que melhor se adequou ao estudo, com um $\mathrm{R}^{2}=0,73$, sendo a regressão cúbica aos 49 DAS a única que obteve efeito significativo com um $\mathrm{R}^{2}$ de 0,91 .

Aos 63 DAS, a TCA das plantas irrigadas com a água de maior salinidade foi superior à TCA do nível de menor salinidade demonstrando atraso no crescimento das plantas irrigadas com a CEa de 2,41 dS m (Tabela 3).

Farias et al. (2003), trabalhando com dois níveis de salinidade e seis lâminas de irrigação, obtiveram TCA média nos intervalos de 19-26, 26-34, 34-40, 40-50 e 50-62, de 1,46, 3,46, 9,65, 8,93 e 6,12 $\mathrm{g} \mathrm{dia}^{-1}$, respectivamente para a cultivar Gold Mine, valores esses diferentes dos obtidos neste estudo, podendo estar relacionados às diferentes variedades e condições de manejo entre os estudos.

Quanto à TCR, verificou-se efeito significativo $(\mathrm{p}<0,01)$ para as doses de $\mathrm{K}_{2} \mathrm{O}$ e sua interação com a salinidade para os períodos de 22 a 28, de 29 a 35 e de 50 a 63 dias após a semeadura (DAS); entre 36 e 49 DAS não houve efeito em relação a doses, porém, foi significativo $(\mathrm{p}<0,05)$ a interação entre doses de K e salinidade (Tabela 3). Em se tratando do efeito da salinidade, encontraram-se efeitos ( $\mathrm{p}<0,01$ ) e $(p<0,05)$ respectivamente, para os intervalos de 22 a 28 
e de 36 a 49 DAS, enquanto em outros intervalos avaliados não houve significância.

A média da TCR, no nível menos salino, em relação à salinidade das águas de irrigação, no intervalo de 21 a 28 DAS, foi superior à da água de CEa = 2,41 dS m-1 Porém, em relação aos outros intervalos de tempo, obteve-se maior taxa de crescimento relativo irrigando-se as plantas com água de maior salinidade (Tabela 3); portanto, a eficiência média para produção de material novo, por unidade de material préexistente, foi melhorada com o incremento da salinidade.

O maior valor de TCR, entre 21 e 28 DAS, de 0,890 $\mathrm{g} \mathrm{g}^{-1} \mathrm{~d}^{-1}$, foi constatado no menor nível salino, combinado com a dose de $273 \mathrm{~kg} \mathrm{ha}^{-1}$ de $\mathrm{K}_{2} \mathrm{O}$, enquanto o menor valor de $0,345 \mathrm{~g} \mathrm{~g}^{-1} \mathrm{~d}^{-1}$, no mesmo intervalo de tempo, ocorreu no maior nível salino com a maior dose de $\mathrm{K}_{2} \mathrm{O}$; com o uso de água mais salina $\left(2,41 \mathrm{dS} \mathrm{m}^{-1}\right)$, verificou-se na dose de $273 \mathrm{~kg} \mathrm{ha}^{-1}$ de $\mathrm{K}_{2} \mathrm{O}$ o maior valor de TCR (fitomassa produzida em relação à matéria seca já existente), com o valor de $0,715 \mathrm{~g} \mathrm{~g}^{-1} \mathrm{~d}^{-1}$. Exceto na dose de $383 \mathrm{~kg} \mathrm{ha}^{-1}$ houve tendência de diminuição da TCR com o incremento das doses de $\mathrm{K}_{2} \mathrm{O}$, enquanto na TCR, entre 29 e 35 DAS, ocorreu decréscimo na produção de material novo, em relação à avaliação anterior, para o nível menos salino, acontecendo o mesmo com a água de maior salinidade; contudo, neste nível salino, combinado com a maior dose de $\mathrm{K}_{2} \mathrm{O}$, se obteve aumento na produção de fitomassa, passando de 0,345 para 0,450 g g $^{-1} \mathrm{~d}^{-1}$.

Entre os intervalos de 36 a 49 e 50 a 63 DAS, ocorreu apenas decréscimo em relação à fitomassa produzida, a partir de material pré-existente

Com relação ao estudo de regressão (Tabelas 4), a significância foi a $1 \%$ para TCR de 50 a 63 DAS, e a $5 \%$ para a TCR de 22 a 28 e de 36 a 49 DAS nas doses de $\mathrm{K}_{2} \mathrm{O}$ dentro do menor nível de salinidade. Para o nível de maior salinidade só ocorreu significância a 1\% na TCR de 29 a 35 DAS e a $5 \%$ na TCR de 22 a 28 DAS.

Em geral, no início do ciclo de cultivo do meloeiro a taxa de crescimento é lenta, com posterior intensificação, até atingir um período de rápido acúmulo de matéria seca, chegando-se ao final do ciclo, com um ritmo de crescimento bastante reduzido, em relação ao período anterior. Este comportamento também foi observado em outros trabalhos (Souza et al., 2003; Farias et al., 2003; Morais et al., 2004; Silva et al.; 2005; Silva Júnior et al., 2006).

\section{CONCLUSÕES}

1. Até 21 dias após a semeadura a água salina $\left(2,41 \mathrm{dS} \mathrm{m}^{-1}\right)$ proporciona maior crescimento da cv. Goldex quando comparada com a água de baixa salinidade $\left(0,52 \mathrm{dS} \mathrm{m}^{-1}\right)$.

2. A partir do intervalo entre 29 e 35 dias após semeadura (DAS), os valores de crescimento relativo são maiores quando se irriga o meloeiro com água de $\mathrm{CEa}=2,41 \mathrm{dS} \mathrm{m}^{-1}$.

3. Independente das doses de $\mathrm{K}_{2} \mathrm{O}$ e salinidade da água, a taxa de crescimento absoluto da planta é máxima entre 35 e 49 DAS.

4. No fim do ciclo, 63 DAS, a dose de $438 \mathrm{~kg} \mathrm{ha}^{-1}$ de $\mathrm{K}_{2} \mathrm{O}$, combinada com água salina $\left(2,41 \mathrm{dS} \mathrm{m}^{-1}\right)$ é a que mais favorece a acumulação de fitomassa seca total.

\section{AGRADECIMENTOS}

Os autores expressam seus agradecimentos ao CNPq (Conselho Nacional de Desenvolvimento Científico e Tecnológico), pelo auxílio financeiro concedido para a realização do trabalho; à Fazenda Santa Júlia, pela cessão da área e pelo apoio no desenvolvimento do trabalho e à UFERSA (Universidade Federal Rural do Semi-árido) pelo apoio logístico.

\section{LITERATURA CITADA}

Alencar, R. D.; Porto Filho, F. de Q.; Medeiros, J. F. de; Holanda, J. F. de; Porto, V. C. N.; Ferreira Neto, M. Crescimento de cultivares de melão amarelo irrigadas com água salina. Revista Brasileira de Engenharia Agrícola e Ambiental, v.7, n.2, p.221-226, 2003.

Benincasa, M. M. P.. Análise de crescimento de plantas. Jaboticabal: FUNEP, 42p. 1988.

Dutra, I.; Medeiros, J. F. de; Porto Filho, F. de Q.; Costa, M. da C. Determinação do fator de cobertura do melão cultivado sob diferentes lâminas e salinidades da água de irrigação. Revista Brasileira de Engenharia Agrícola e Ambiental, v.4, n.2, p.146-151, 2000.

EMBRAPA - Empresa Brasileira de Pesquisa Agropecuária. Centro Nacional de Pesquisa de Solo. Sistema brasileiro de classificação de solos. Rio de Janeiro: Embrapa Solos. 1999. 412p.

Epstein, E.; Bloom, A. J. Nutrição mineral de plantas: Princípios e perspectivas. Londrina: Planta, 2006. 403p.

Farias, C. H. de. A.; Espínola Sobrinho, J.; Medeiros, J. F. de; Costa, M. da C.; Nascimento, I. B.; Silva, M. C. de C. Crescimento e desenvolvimento da cultura do melão sob diferentes lâminas de irrigação e salinidade da água. Revista Brasileira de Engenharia Agrícola e Ambiental, v.7, n.3, p.445-450, 2003.

Ferreira, D. F. Manual do sistema SISVAR para análises estatísticas. Lavras: UFV, 2000, 66p.

Filgueira, F. A. R. Novo manual de olericultura: Agrotecnologia moderna na produção e comercialização de hortaliças. Viçosa: UFV, 2000. 402p.

Giehl, R. F. H.; Fagan, E. B.; Eisermann, A. C.; Brackmann, A.; Medeiros, S. P.; Manfron, P. A. Crescimento e mudanças físico-quimícas durante a maturação de frutos de meloeiro ( $\mathrm{Cucu}$ mis melo var. cantalupensis Naud.) hibrido torreon. Ciência e Agrotecnologia, v.32, n.2, p.371-377, 2008

Gurgel, M. T.; GheyI, H. R.; Oliveira, F. H. T.; Uyeda, C. A.; Fernandes, P. D.; Almeida Filho, F. D. Análise econômica do uso de água salina no cultivo de meloeiro. Revista Brasileira de Engenharia Agrícola e Ambiental, v.9 (suplemento) p.258-262, 2005.

IBGE - Instituto Brasileiro de Geografia e Estatística. Sistema de recuperação automática - Sidra: Produção agrícola municipal. Quantidade produzida, valor da produção, área plantada, e área colhida da lavoura temporária. http://www.sidra.ibge.gov.br/. 19 Mai. 2009. 
Medeiros, J. F. de; Duarte. S. R.; Fernandes, P. D.; Dias, N. da S.; Gheyi, H. R. Cresceimento e acúmulo de N, P e K pelo meloeiro irrigado com água salina. Horticultura Brasileira, v.26, n.4, p.452-457, 2008.

Morais, E. R. C. de; Maia, C. E.; Lima Júnior, O. J. de; Negreiros, M. Z. de; Medeiros, J. F. de; Espínola Sobrinho, J.; Leitão, M. de M. V. B. R.; Oliveira, F. de A. Crescimento de melão cantaloupe torreon cultivado com diferentes cores de mulch e lâminas de irrigação nas condições de Mossoró, RN. In: Congresso Brasileiro de Olericultura, Horticultura Brasileira, 44, 2004, Campo Grande. Anais... Brasília: ABH, 2004. CD-Rom

Oliveira, M.; Maia C. E. Qualidade físico-química da água para irrigação em diferentes aqüíferos na área sedimentar do Estado do Rio Grande do Norte. Revista Brasileira de Engenharia Agrícola e Ambiental, v.2, p.42-46, 1998.

Porto Filho, F. de Q.; Medeiros, J. F.; Gheyi, H. R.; Matos, J. de A., de; Souza, E. R.; Sousa Neto, E. R. Crescimento do meloeiro irrigado com águas de diferentes salinidades. Horticultura Brasileira, v.24, p.334-341, 2006a.

Porto Filho, F. de. Q.; Medeiros, J. F. de; Sousa Neto, E. R. de; Gheyi, H. R.; Matos, J. de A. de. Viabilidade da irrigação do meloeiro com águas salinas em diferentes fases fenológicas. Ciência Rural, v.36, n.2, p.453-459, 2006b

Queiroga, R. C. F. de; Andrade Neto, R. de C.; Nunes, G. H de S.; Medeiros, J. F. de; Araújo, W. de B. M. de. Germinação e crescimento inicial de híbridos de meloeiro em função da salinidade. Horticultura Brasileira, v.24, n.3, p.315-319, 2006.
Satti, S. M. E.; Lopez, M. Effect of increasing potassium levels for alleviating sodium chloride stress on the growth and yield of tomato. Communications Soil Science and Plant Analysis, v.25, n.15-16, p.2807-2823, 1994.

Silva, B. B. da; Ferreira, J. A. da S.; Rao, T. V. R.; Silva, V. de P. R. da. Características de parâmetros fisiológicos e de crescimento do meloeiro irrigado. Revista Brasileira de Agrometeorologia, v.13, n.1, p.45-52, 2005.

Silva Junior, M. J. da; Medeiros, J. F. de; Oliveira, F. H. T. de; Dutra, I. Acúmulo de matéria seca e absorção de nutrientes pelo meloeiro "pele-de-sapo”. Revista Brasileira de Engenharia Agrícola e Ambiental, v.10, n.2, p.364-368, 2006.

Silva Junior, M. J. da; Medeiros, J. F. de; Oliveira, F. H. T. de; Dutra, I. Balanço da fertirrigação em meloeiro "pele-de-sapo”. Irriga, v.12, n.1, p.63-72, 2007.

Souza, E. R. de; Levien, S. L. A.; Medeiros, J. F. de; Porto Filho, F. de Q.; Gheyi, H. R.; Sousa Neto, E. R de; Silva Junior, M. J. da. Crescimento do meloeiro (Cucumis melo L.) submetido a diferentes níveis de salinidade de água em fases fenológicas distintas. Caatinga, v.16, n.1/2, p.31-38, 2003.

Tester, M.; Davenport, R. Na+ Tolerance and $\mathrm{Na}^{+}$transport in higher plants. Annals of Botany, v.91, n.5, p.503-527, 2003.

Uyeda, C. A.; Gurgel, M. T.; Gheyi, H. R.; Oliveira, F. H. T.; Fernandes, P. D.; Produtividade de duas cultivares de meloeiro irrigadas com água de alta e baixa salinidade. Revista Brasileira de Engenharia Agrícola e Ambiental, suplemento, v.9 (suplemento), p.318-321, 2005. 